\title{
The evolution of HPV-related anogenital cancers reported in Quebec - Incidence rates and survival probabilities
}

\author{
R Louchini, MSc; P Goggin, MD, MSc; M Steben, MD (1)
}

\begin{abstract}
Non-cervical anogenital cancers (i.e. anal, vulvar, vaginal and penile cancers) associated with the human papillomavirus (HPV), for which HPV is known to be the necessary cause of carcinogenesis, are poorly documented due to their relatively low incidence rate. The aim of this study is to describe the incidence rates of these cancers between 1984 and 2001, and their relative survival probabilities, in Quebec (Canada) between 1984 and 1998. The incidence of these cancers is on the rise, particularly anal cancer in women and, more recently (since 1993-95), vulvar cancer. Between 1984-86 and 1993-95, the 5-year relative survival probability for men with anal cancer decreased from $57 \%$ to $46 \%$, while that for penile cancer dropped from $75 \%$ to 59\%. However, during the same period, the 5-year relative survival probability for women with anal cancer rose from $56 \%$ to $65 \%$, and remained stable for cervical and vulvar cancers, at $74 \%$ and $82 \%$, respectively.
\end{abstract}

Key words: alphapapillomavirus, uterine cervical neoplasm, anal neoplasm, vaginal neoplasm, vulvar neoplasm, penile neoplasm, survival probability

\section{Introduction}

The fight against the human papillomavirus (HPV) has taken a major turn since this infection was identified as the necessary cause of cervical cancer. In fact, in addition to including HPV-detection tests in the screening or follow-up of cytological anomalies, the battle against this disease has been bolstered by the development of prophylactic vaccines. ${ }^{(1)}$

HPV is associated with a spectrum of diseases other than cervical cancer. It is now believed to be associated with other anogenital cancers, including over $80 \%$ of anal cancers and approximately $40 \%$ to $50 \%$ of penile, vulvar and vaginal cancers. $^{(2,3)}$ It is also associated with a significant proportion of oropharyngeal cancers, ${ }^{(2,3)}$ and may be associated with other cancers ${ }^{(4)}$ as well.
To date, non-cervical anogenital cancers associated with HPV have been poorly documented due to their low incidence rate. A few studies have reported an increase in the incidence rate of anal cancer in the U.S., ${ }^{(5-7)}$ Sweden $^{(8)}$ and Denmark $^{(9)}$ over the past several decades; however, population data are scarce for the other types of anogenital cancers (vulvar, vaginal, penile).$^{(10-12)}$

The aim of this study is to describe the significance and evolution of HPVrelated anogenital cancers in Quebec. More specifically, this epidemiological portrait will address the incidence rates of cervical, anal, vulvar, vaginal and penile cancers in Quebec from 1984 to 2001, as well as the relative survival probabilities associated with these cancers from 1984 to 1998 . The evolution of the incidence rate of cancer may provide insight into the changes in causal risk factors. It may also help evaluate the effects of screening, diagnostic and prevention procedures for these types of cancers.

\section{Method}

\section{Data source}

The data on the incidence rate of cancers diagnosed in Quebec between 1984 and 2001, inclusively, were taken from the Fichier des tumeurs du Québec (FiTQ) [Quebec tumour registry]. The population data used to calculate the rates are Institut de la statistique du Québec (ISQ) [Quebec statistics centre] estimates, based on national censuses (Statistics Canada) and corrected to account for underenumeration, over-enumeration and nonpermanent residents.

With respect to the calculation of survival probabilities, the vital status of individuals whose cancers were reported to the FiTQ between 1984 and 1998 was determined by linking their case reports with the Quebec death registry from 1984 to 1999 , inclusively. The linking of these two registries is described in the report entitled "Cancer survival of newly diagnosed cases, Quebec, 1992" ${ }^{(13)}$ in the section on the quality of data used. The cancers addressed in this study are anal (ICD-9 154.2, 154.3 and 154.8), cervical (ICD-9 180), vaginal (ICD-9 184.0), vulvar (ICD-9 184.1, 184.2, 184.3 and 184.4) and penile cancers (ICD-9 187.1, 187.2, 187.3 and 187.4, 187.7 and 187.8).

1 Institut national de santé publique du Québec, QC

Correspondence: Rabiâ Louchini, Institut national de santé publique du Québec, 945 Wolfe Ave., 2nd floor, Québec, QC, Canada G1V 5B3, Tel: (418) 650-5115, ext. 5242, Fax: (418) 654-3132, e-mail: rabia.louchini@inspq.qc.ca 


\section{Calculation of standardized incidence rates}

The reference population used to calculate the standardized incidence rates was that of the 1991 Canadian census, distributed by age group, both sexes combined. For each type of cancer, the incidence rate was calculated based on three categories of morphology (squamous-cell, adenocarcinoma and other). Squamouscell cancers are the most frequent (67\%, $58 \%, 64 \%, 57 \%$ and $74 \%$, respectively, for cancers of the vulva, vagina, cervix, anus and penis).

\section{Calculation of survival probabilities}

The calculation of survival probabilities was based on newly diagnosed cases of cancer reported to the Quebec tumour registry, involving individuals under 100 years of age at diagnosis. Only the first primary cancer reported in an individual between 1984 and 1998, inclusively, was retained. Subsequent primary cancers were excluded.

The relative survival probability is a ratio of the probability of survival observed in a group of patients to the expected probability of survival of a comparable group within the general population that, hypothetically, does not have the cancer being studied and has the same demographic characteristics as the group of cancer patients. The observed survival probability is calculated according to the actuarial method, i.e. the ratio of the number of survivors at the end of the follow-up period to the number of living individuals at the beginning of that same period. The expected survival probability was estimated using three mortality tables for the general population of Quebec, ${ }^{(14-16)}$ based on the Ederer II method. ${ }^{(17)}$ The calculation of the relative survival probability is described in detail by Louchini et al. ${ }^{(18)}$

\section{Findlings}

\section{Incidence rates of and relative survival probabilities for anogenital cancers}

From 1984 to 2001, there were 9556 reported cases of primary anogenital cancers, all types combined: 988 (10\%) in men and 8568 (90\%) in women. This large proportion observed in women is attributable mainly to cases of cervical cancer, which alone represents $65 \%$ of all anogenital cancers. Table 1 presents the distribution of these cancers by site, sex and time period. Figure 1 presents this distribution by site, sex and age, for the entire period. With the exception of cervical cancer, which is most common in women aged 40-59, anogenital cancers are most common in the 60-79 age group, which accounts for roughly $50 \%$ of all such cancers observed.

Cervical cancer has the highest incidence rate among all anogenital cancers, followed by vulvar cancer. For example, between 1999 and 2001, the incidence rate was 5.6 per 100000 women for cervical cancer and 1.3 per 100000 women for vulvar cancer. Incidence rates for the other cancers range from 0.2 to 0.8 per 100000 (Figure 2).

A total of 7201 cases of anogenital cancer reported as the first primary cancer were used to calculate the relative survival probabilities between 1984 and 1998. They are distri-buted as follows: 4822 cases of cervical cancer, 900 cases of vulvar cancer, 290 cases of vaginal cancer, 360 cases of penile cancer and 759 cases of anal cancer (338 men and 421 women). Relative survival probabilities vary by site and sex. The 5-year relative survival probability is highest for vulvar cancer (81\%) followed by cervical cancer $(72 \%)$, penile cancer $(67 \%)$, anal cancer $(53 \%$ in men and $60 \%$ in women) and, finally, vaginal cancer (49\%). Figure 3 presents the relative survival probabilities for each site, up to 15 years post-diagnosis. While relative survival probabilities for vulvar cancer and vaginal cancer have reached a plateau at 6 years and 9 years postdiagnosis, respectively, those for penile cancer and anal cancer have decreased to $59 \%$ and $42 \%$ at 15 years post-diagnosis, respectively.

Relative survival probabilities also vary by age group, decreasing with age for most sites (Figure 4); however, the relative survival profiles for vaginal cancer, anal cancer in men and penile cancer are different for the youngest age group. In fact, the relative survival probability for this age

[cont'd, p. 104]

TABLE 1

Distribution of the number of new cases (\% in parentheses) of cancer, by site (all morphologies), sex, and time period, Quebec, 1984-1986 to 1999-2001

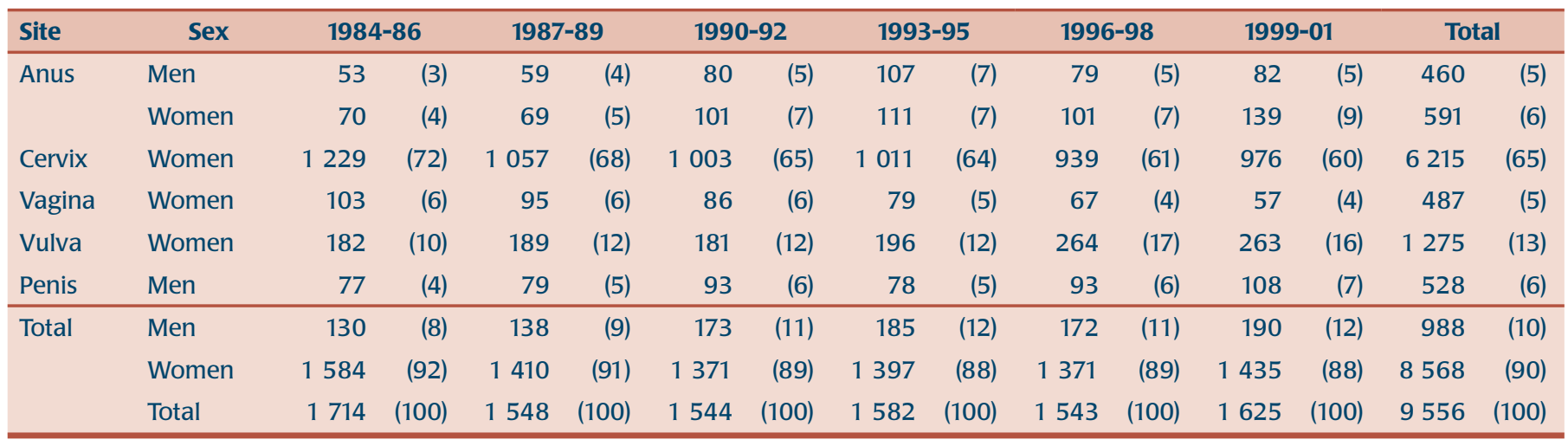


FIGURE 1

Distribution of new cases of cancer, by site (all morphologies), sex and age, Quebec, 1984-2001

a) Anal cancer in men

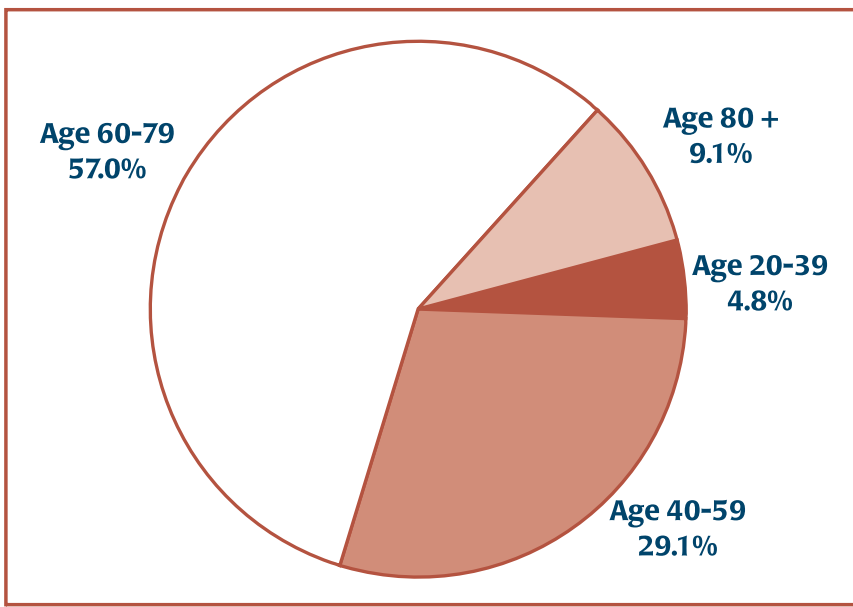

\section{c) Cervical cancer}

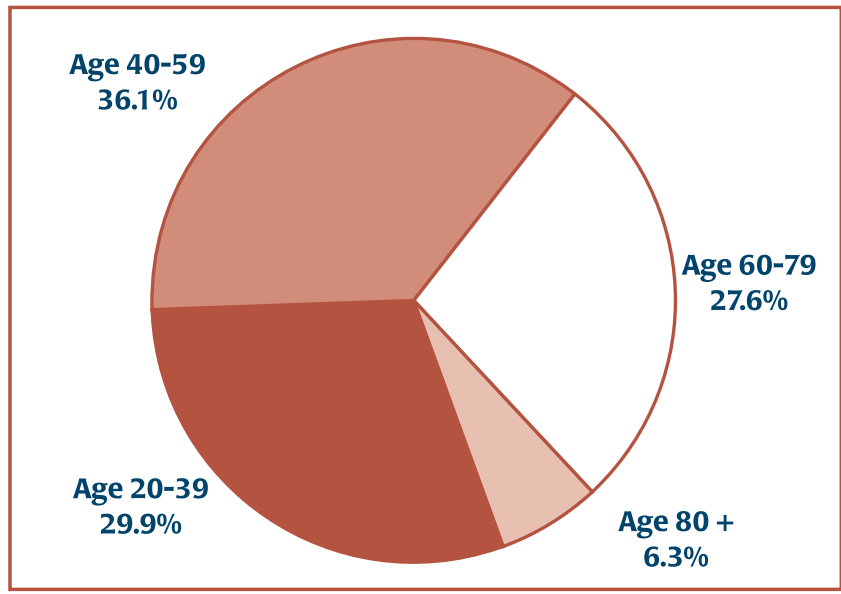

\section{e) Vulvar cancer}

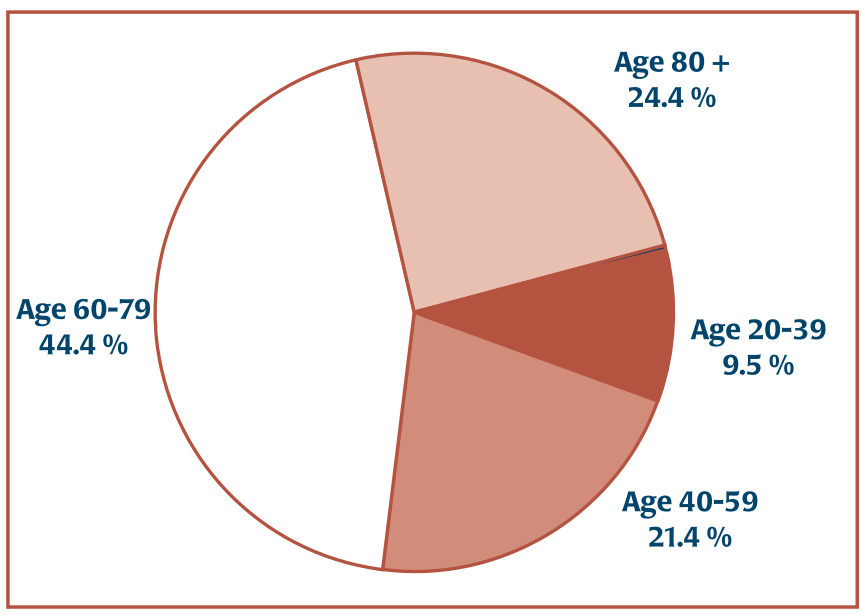

b) Anal cancer in women

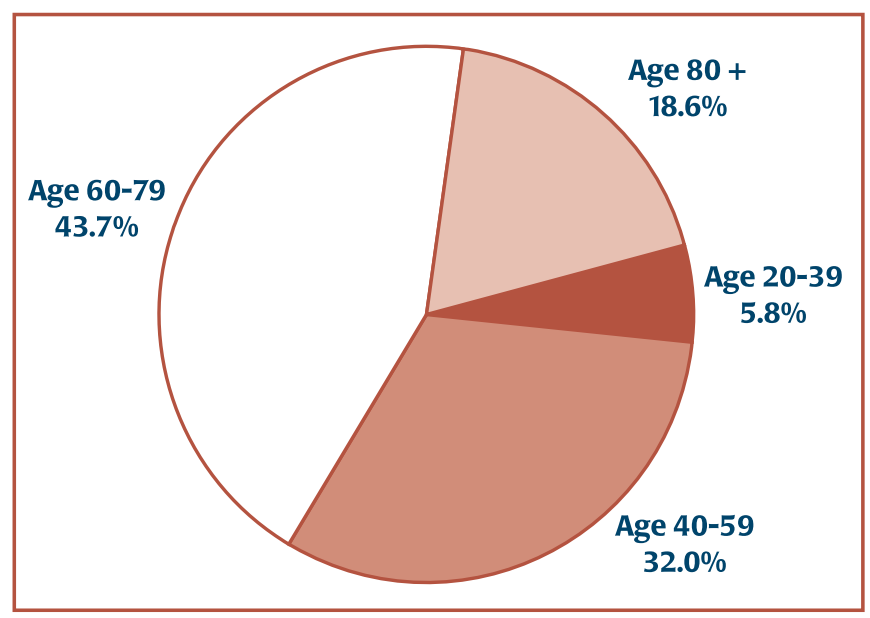

d) Vaginal cancer

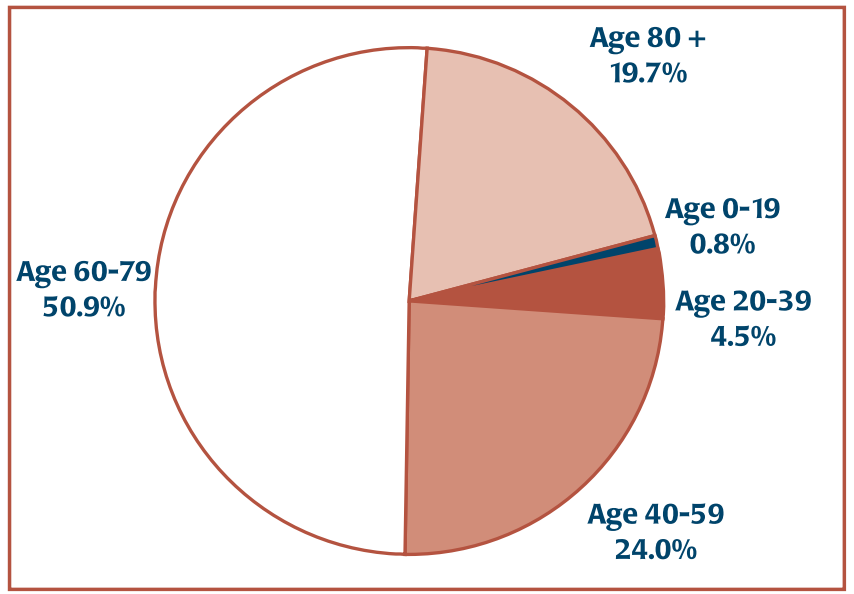

\section{f) Penile cancer}

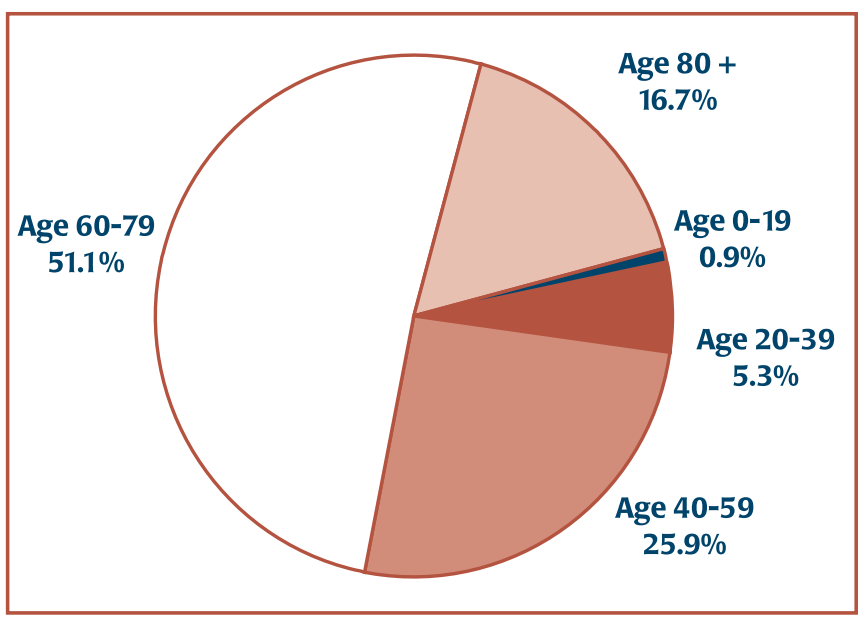


FIGURE 2

Evolution of standardized incidence rates of anogenital cancers, by sex, Quebec, from 1984-1986 to 1999-2001

\section{a) Anal cancer in men}

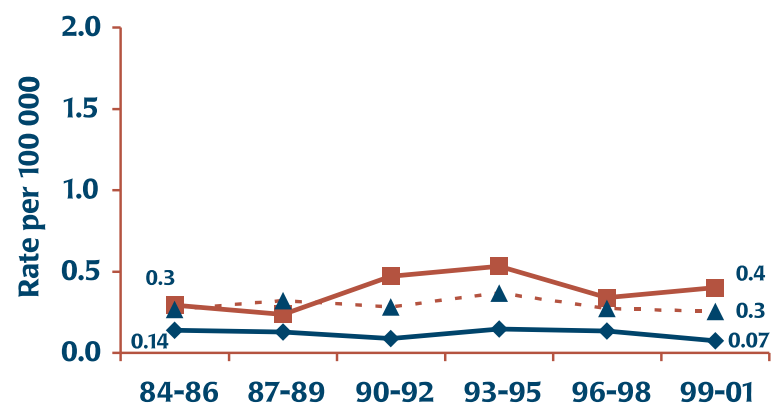

Year

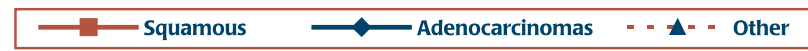

\section{c) Cervical cancer}

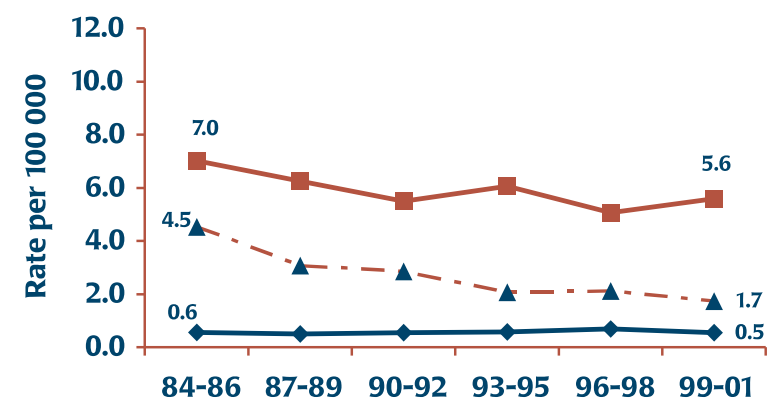

Year

$\longrightarrow$ Squamous $\longrightarrow$ Adenocarcinomas $-\Lambda-\cdot$ Other

e) Vulvar cancer

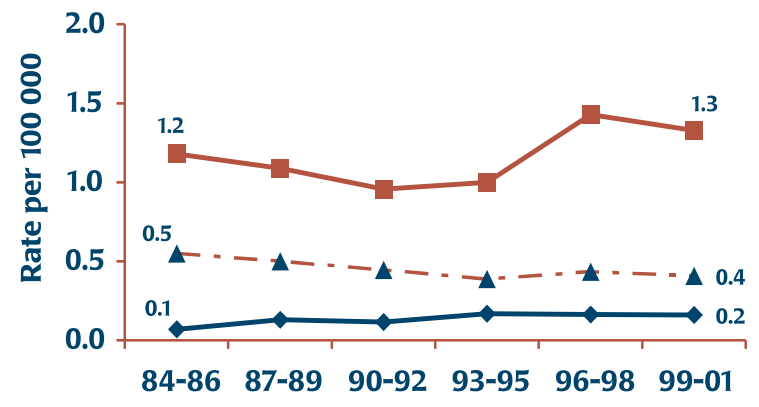

Year

$\longrightarrow$ Squamous $\longrightarrow$ Adenocarcinomas $-\mathbf{A}-=$ Other

b) Anal cancer in women

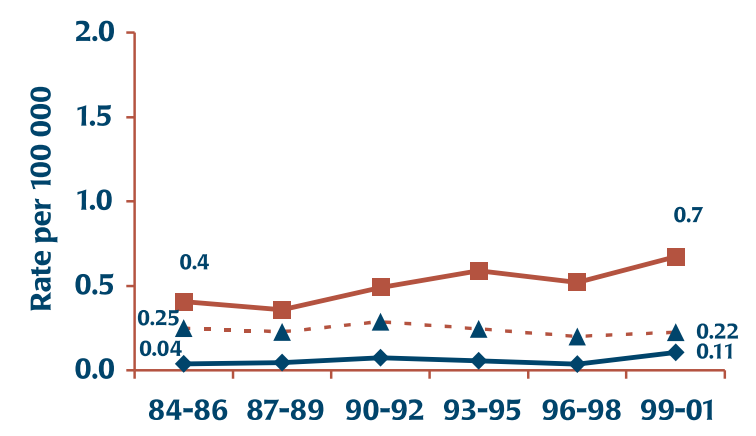

Year

$\longrightarrow$ Squamous $\longrightarrow$ Adenocarcinomas $=-\mathbf{A}^{*}=$ Other

d) Vaginal cancer
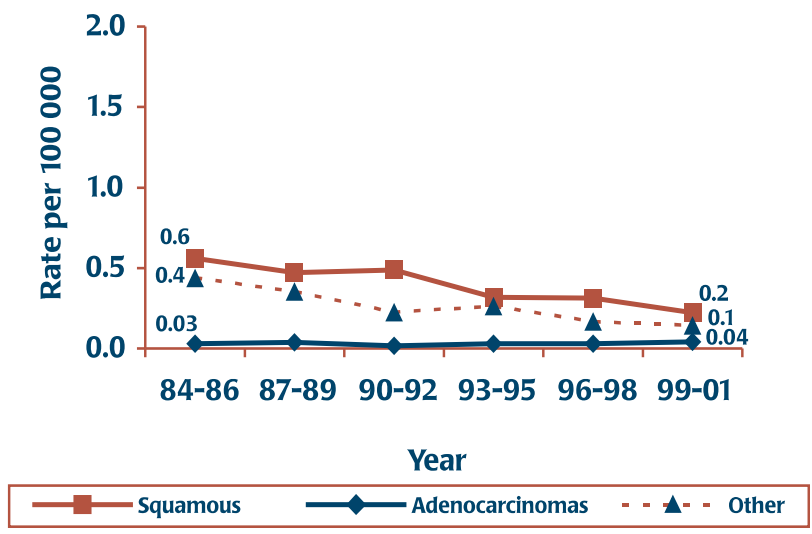

f) Penile cancer

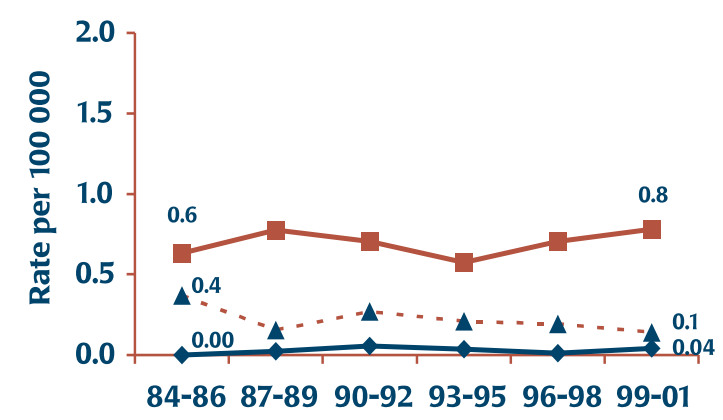

Year

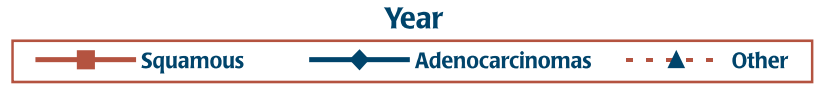


FIGURE 3

Relative survival (\%), by site (all morphologies) and sex, Quebec, 1984-1998

a) Anal cancer in men

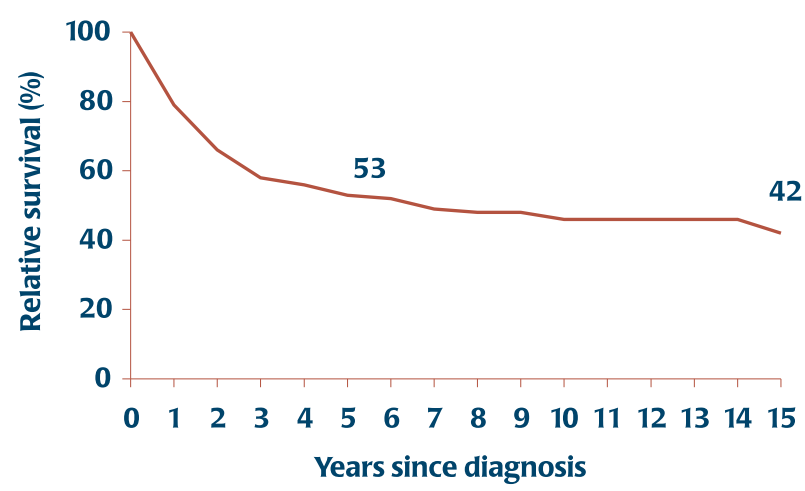

c) Cervical cancer

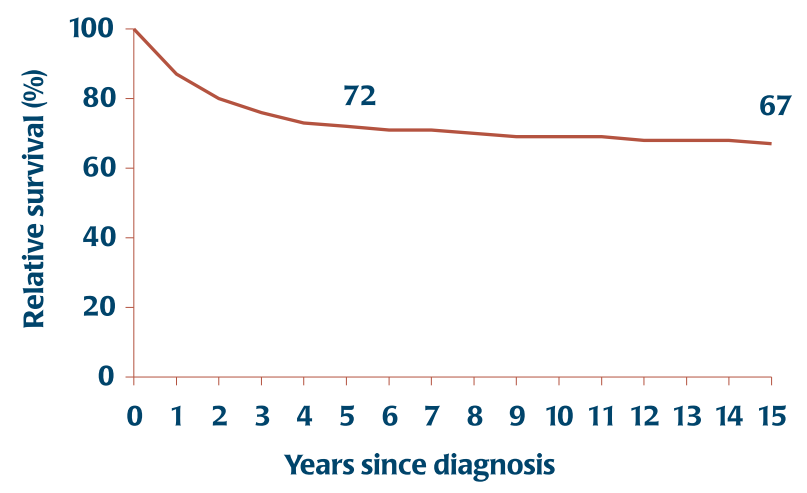

e) Vulvar cancer

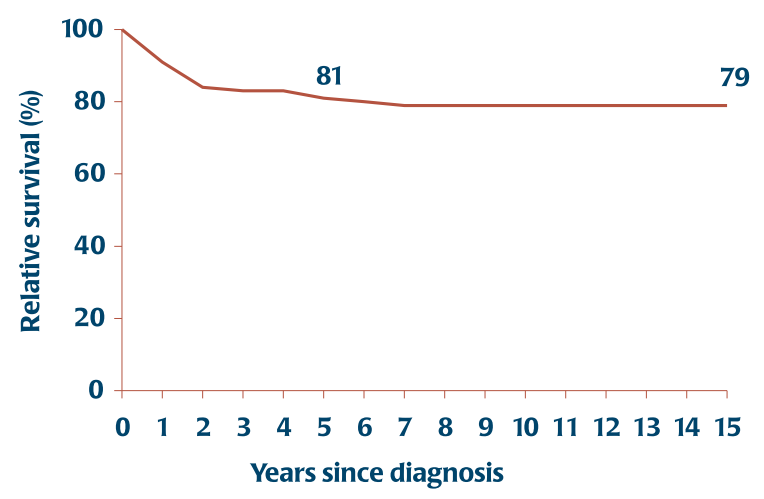

\section{b) Anal cancer in women}

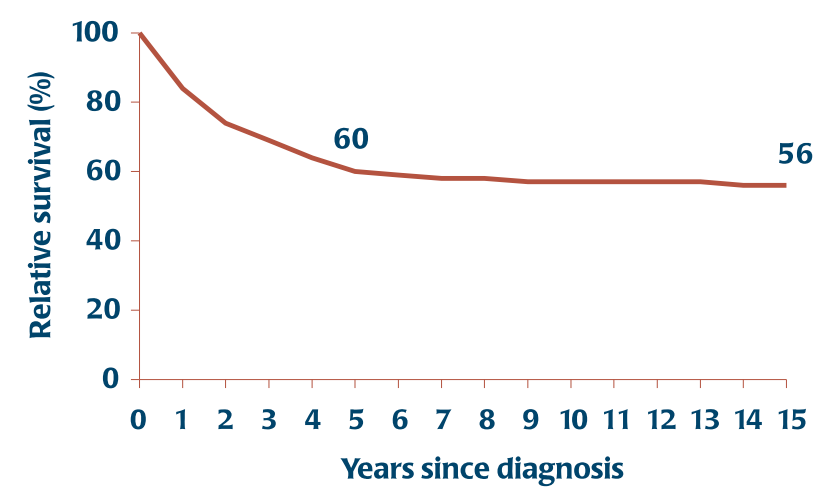

d) Vaginal cancer

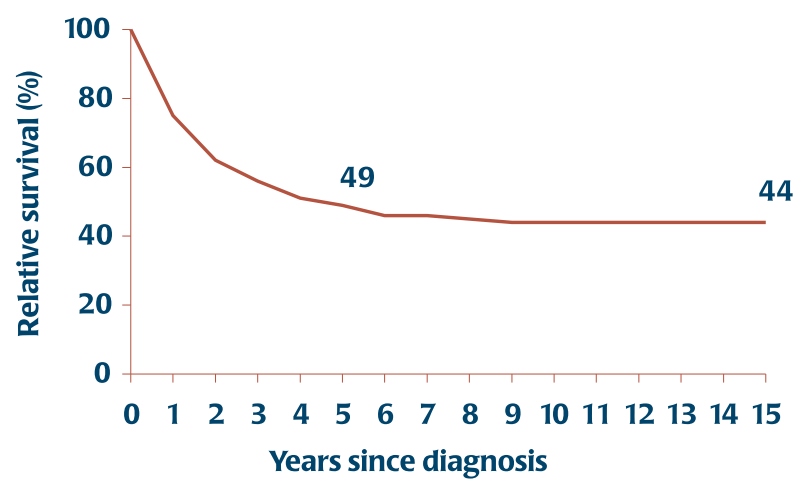

f) Penile cancer

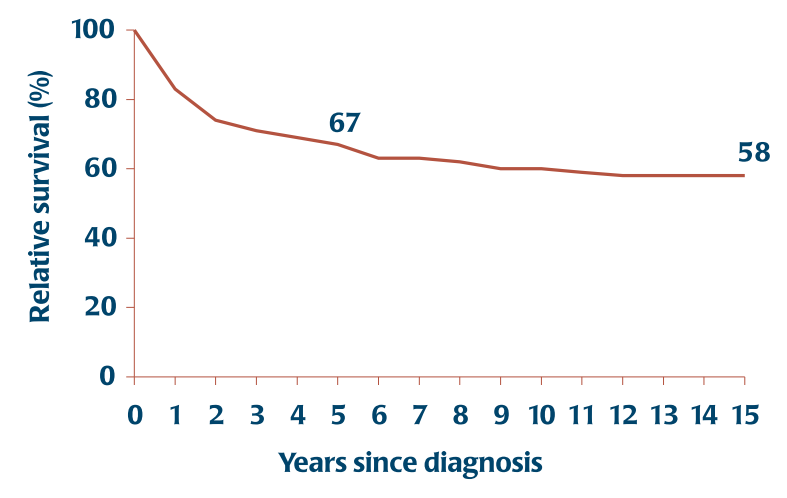


FIGURE 4

Five-year relative survival (\%), by age group and site (all morphologies), Quebec, 1984-1995

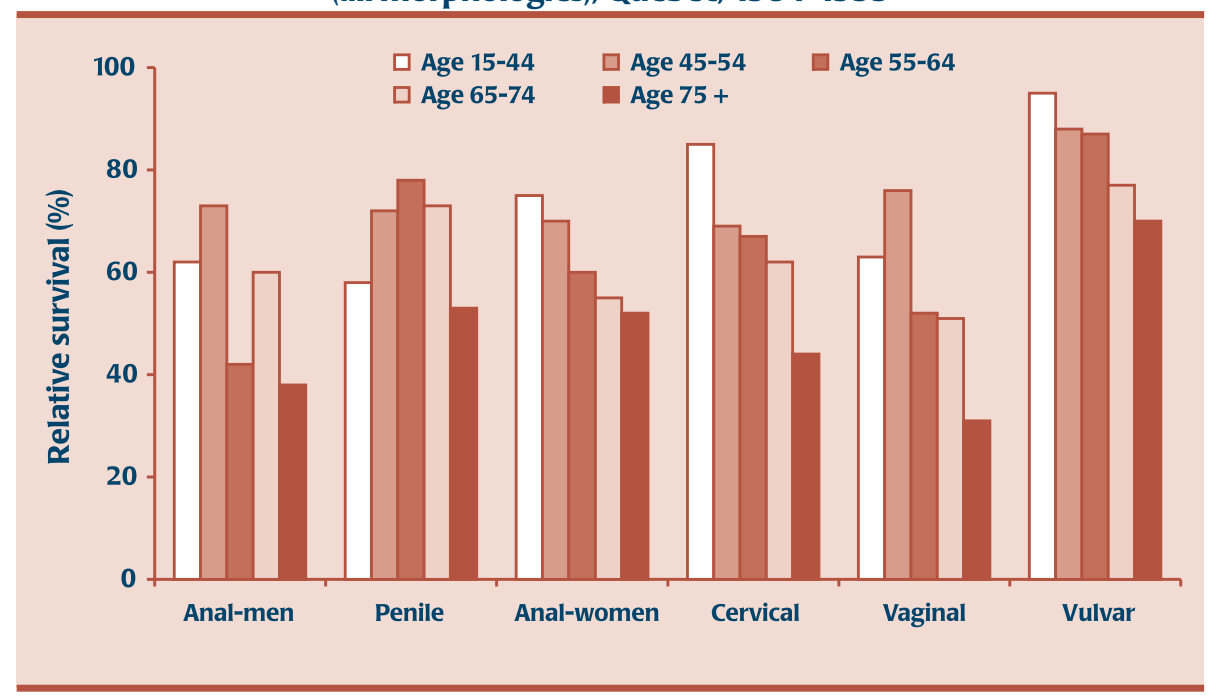

group is not the highest, as compared with the other anogenital cancer sites.

been a decline in the incidence of cervical and vaginal cancers.

\section{Evolution of the incidence of ano- genital cancers}

The incidence rate of anal cancer increased between 1984-1986 and 1999-2001, particularly in women (Figure 2), and the incidence of vulvar cancer has increased slightly since 1993-95. However, there has

\section{Evolution of the relative survival probabilities for anogenital cancers}

The relative survival probabilities for men with anal or penile cancer dropped from $57 \%$ and $75 \%$, respectively, in $1984-86$, to $46 \%$ and $59 \%$ in $1993-95$. However, the 5-year relative survival

FIGURE 5

Five-year relative survival (\%), by diagnosis period and site (all morphologies), Quebec, 1984-1986 to 1993-1995

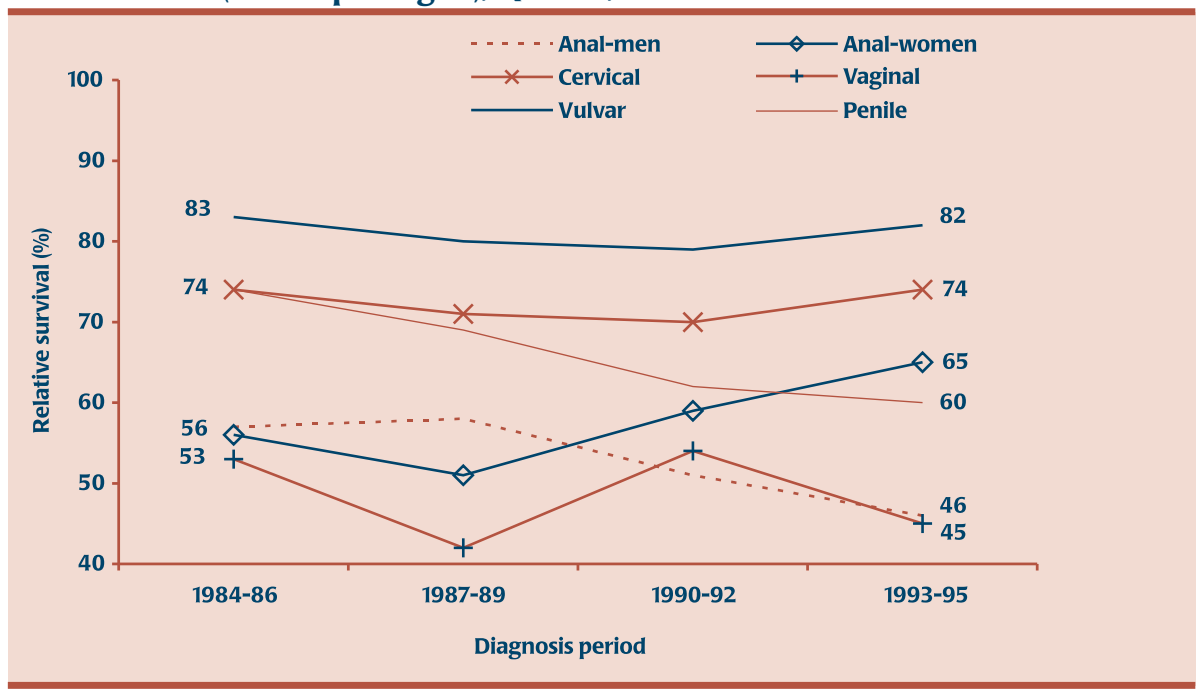

probability for women with anal cancer increased from $56 \%$ to $65 \%$. The 5 -year survival probability for cervical and vulvar cancers remained stable at $74 \%$ and $82 \%$, respectively (Figure 5).

\section{Relationship between the incidence of anal cancer and cervical cancer in women in Quebec between 1984 and 1999: incidence rate of a second cancer site}

We also studied the relationship between anal cancer and cervical cancer in women. Only 3 women in 5818 who had either cervical cancer or anal cancer also had a second cancer at a different site (cervix or anus) during the 17-year observation period in Quebec.

\section{Discussion}

The results of this study show that, between 1984 and 2001, the incidence of anogenital cancers in Quebec, particularly anal cancer in women and vulvar cancer since 1993-95, was low but rising. The same trend was observed for anal cancer in several countries, specifically the U.S., Denmark and Sweden, ${ }^{(5,6,8,9,19,20)}$ and for vulvar cancer in Sweden ${ }^{(21)}$ between 195869 and 1990-96 and in Austria ${ }^{(22)}$ between 1985-88 and 1994-97. However, the latter increase was not observed in Norway, Switzerland or the U.S. ${ }^{(11,23,24)}$ The surge in vulvar intraepithelial neoplasia (VIN), a precursor to vulvar cancer, ${ }^{(24)}$ particularly in young women, ${ }^{(25)}$ may explain the observed increase in the incidence of vulvar cancer. A New Zealand study reported that $87.5 \%$ of 113 untreated cases of grade- 3 VIN evolved into invasive cancer. ${ }^{(26)}$

In contrast to other anogenital cancers, the incidence rates of cervical cancer and vaginal cancer dropped markedly. This drop in the incidence of vaginal cancer was also observed in Switzerland. In fact, the incidence rate of vaginal cancer (per 100 000) dropped from 0.8 in 1974-84 to 0.4 in 1985-94; ${ }^{(11)}$ conversely, a slight increase was observed in Sweden. ${ }^{(21)}$ Early screening with the introduction of the Pap test in 
the 1960s accounts for the reduction in the number of cervical cancers. Similarly, the withdrawal of diethylstilbestrol (DES) from the market in 1971 may also explain the lower incidence of cervical and vaginal cancers. DES, a synthetic form of estrogen, was found to be associated with a higher risk of squamous intraepithelial neoplasia and clear cell adenocarcinoma of the cervix and vagina in girls whose mothers were treated with this drug during pregnancy. ${ }^{(27)}$ This hormone was prescribed between 1941 and 1971 to women who experienced bleeding during pregnancy, had had previous miscarriages or were diabetic. ${ }^{(28)}$

Genital HPV infection raises the issue of the risk of multiple cancers, particularly anal cancer in women and cervical cancer. In Quebec, only 3 women had both anal cancer and cervical cancer over a 17-year study period. While this result does not enable us to establish a clear link between these two cancer sites, a retrospective study measuring the standardized incidence ratios of low- and high-grade cervical intraepithelial neoplasia (CIN 1, 2 and 3) and the incidence of concomitant and subsequent anogenital cancers (cervical, anal, vulvar and vaginal) indicates a strong relationship. ${ }^{(29)}$

The findings also indicate that the relative survival probability is lower in the youngest age group for anal cancer in men and for penile and vaginal cancers. Contrasting results for anal cancer were reported in 2006 in a study conducted by Jeffreys et al. in England and Wales. ${ }^{(30)}$ Moreover, our study revealed a marked difference between men and women in the relative survival probability for anal cancer, a gap which continued to widen, reaching $19 \%$ in 1993-95. Whereas the relative survival probability increased for women, it decreased for men. The same trend was observed in the U.S.; ${ }^{(7)}$ however, in England and Wales, the 5-year survival probability for men with anal cancer improved between 1986 and 2001. ${ }^{(30)}$

Anal cancer is more common in men who have receptive anal sex and who are infected with HIV. ${ }^{(9)}$ A 2005 Quebec study conducted by Goggin et al. found that the incidence rate of squamous-cell anal cancer in men had risen dramatically in the Montreal area. ${ }^{(31)}$ In fact, between 1984 and 2001, the incidence of squamous-cell anal cancers in the metropolitan Montreal area rose by $353 \%$ in men and $134 \%$ in women; however, no increase in the incidence of anal adenocarcinoma-representing approximately $21 \%$ of caseswas observed during the same period. Antiretroviral therapy can prolong the life expectancy of HIV-positive individuals with chronic immunodeficiency, a condition that promotes the development of anal cancer, thereby increasing its incidence. However, these individuals risk having a poor prognosis following their diagnosis of cancer, which could partly explain the low survival probability observed for these cancers.

Secondary prevention through early screening of precancerous cervical lesions and primary prevention through vaccination are the best courses of action to reduce the burden of this disease by reducing its incidence. Generally speaking, once at the cancer stage, the efficacy of treatment seems to reach a plateau at which point mortality no longer decreases. Moreover, in Quebec, the relative survival probability, an indicator of the efficacy of treatment, has remained unchanged over the years for both cervical cancer and the majority of the main cancer sites. ${ }^{(18)}$

In conclusion, although in Quebec the number of cases of anogenital cancers is relatively low compared to other cancer sites, it is continuously on the rise, and the associated survival probability is mediocre in men and is continuously decreasing. HPV continues to represent a heavy burden. The development of prophylactic HPV vaccines is a primary prevention measure which, when combined with screening as a secondary prevention measure (in the case of cervical cancer), should provide protection against HPV.

\section{Acknowledgements}

We would like to thank Michel Beaupré of the Ministère de la Santé et des Services sociaux, Bernard Duval of the Institut national de santé publique du Québec and Alain Demers of CancerCare Manitoba for their insightful comments in finalizing this document.

\section{References}

1. Franco EL, Harper DM. Vaccination against human papillomavirus infection: a new paradigm in cervical cancer control. Vaccine 2005;23:2388-94.

2. Parkin DM, Bray F. Chapter 2: The burden of HPV-related cancers. Vaccine 2006;24 Suppl 3:S11-S25.

3. Spence AR, Franco EL, Ferenczy A. The Role of Human Papillomaviruses in Cancer. Evidence to Date. Am J Cancer 2005;4:49-64.

4. Gillison ML, Shah KV. Chapter 9: Role of mucosal human papillomavirus in nongenital cancers. J Natl Cancer Inst Monographs 2003;57-65.

5. Melbye M, et al. Changing patterns of anal cancer incidence in the United States, 19401989. Am J Epidemiol 1994;139:772-80.

6. Maggard MA, Beanes SR, Ko CY. Anal canal cancer: a population-based reappraisal. Dis Colon Rectum 2003;46:1517-23.

7. Johnson LG, et al. Anal cancer incidence and survival: the surveillance, epidemiology, and end results experience, 19732000. Cancer 2004;101:281-8.

8. Goldman S, et al. Incidence of anal epidermoid carcinoma in Sweden 19701984. Acta chirurgica Scandinavica 1989; 155:191-7.

9. Frisch M, Melbye M, Moller H. Trends in incidence of anal cancer in Denmark. BMJ (Clinical research ed.) 1993;306:419-22.

10. Cheon J, et al. Survey of incidence of urological cancer in South Korea: a 15-year summary. Int J Urol 2002;9:445-54.

11. Levi F, Randimbison L, La Vecchia C. Descriptive epidemiology of vulvar and vaginal cancers in Vaud, Switzerland, 1974-1994. Ann Oncol 1998;9:1229-32. 
12. Maiche AG. Epidemiological aspects of cancer of the penis in Finland. Eur J Cancer Prev 1992;1:153-8.

13. Louchini R. Cancer survival of newly diagnosed cases, 1992: Data quality, fiveyear relative survival rates. Montreal: Institut national de santé publique, Direction du développement et des programmes, Unité connaissance-surveillance, 2002.

14. Duchesne D, et al. Life tables, Canada, provinces and territoies, 1995-1997. Ottawa: Statistics Canada, 2002.

15. Millar WJ, David P. Life tables, Canada and provinces, 1990-1992. Ottawa: Statistics Canada, 1995.

16. Statistics Canada. Life tables, Canada and provinces, 1986. Ottawa: Statistics Canada, 1990.

17. Ederer F, Heise H. Instructions to IBM 650 programmers in processing survival computations. Methodological note No.10, End Results Evaluation Section. 1959. National Cancer Institute, Bethesda MD.

18. Louchini R, Beaupré M. Survival probabilities for cancer cases reported between 1984 and 1998 in Quebec: Observed \& relative survival. 2003.

19. Cress RD, Holly EA. Incidence of anal cancer in California: increased incidence among men in San Francisco, 1973-1999. Prev Med 2003;36:555-60.
20. Diamond C, et al. Increased incidence of squamous cell anal cancer among men with AIDS in the era of highly active antiretroviral therapy. Sex Transm Dis 2005;32:314-20.

21. Hemminki $\mathrm{K}$, Li $\mathrm{X}$, Vaittinen P. Time trends in the incidence of cervical and other genital squamous cell carcinomas and adenocarcinomas in Sweden, 19581996. Eur J Obstet Gynecol Reprod Biol 2002;101:64-9.

22. Joura EA, et al. Trends in vulvar neoplasia. Increasing incidence of vulvarintraepithelial neoplasia and squamous cell carcinoma of the vulva in young women. J Reprod Med 2000;45:613-5

23. Sturgeon SR, et al. In situ and invasivevulvar cancer incidence trends (1973 to 1987). Am J Obstet Gynecol 1992;166:1482-5.

24. Iversen $\mathrm{T}$, Tretli $\mathrm{S}$. Intraepithelial and invasive squamous cell neoplasia of the vulva: trends in incidence, recurrence, and survival rate in Norway. Obstet Gynecol 1998;91:969-72.

25. Jones RW, Baranyai J, Stables, S. Trends in squamous cell carcinoma of the vulva: the influence of vulvar intraepithelial neoplasia. Obstet Gynecol 1997;90:448-52.
26. Jones RW, Rowan DM. Vulvar intraepithelial neoplasia III: a clinical study of the outcome in 113 cases with relation to the later development of invasive vulvar carcinoma. Obstet Gynecol 1994;84:741-5.

27. Hatch EE, et al. Incidence of squamous neoplasia of the cervix and vagina in women exposed prenatally to diethylstilbestrol (United States). Cancer Causes Control 2001;12:837-45.

28. D.E.S. Action Canada. D.E.S. http://www. web.net/ desact/anglais/anglais.html. 2007.

29. Kalliala I, et al. Risk of cervical and other cancers after treatment of cervical intraepithelial neoplasia: retrospective cohort study. BMJ (Clinical research ed.) 2005;331:1183-5.

30. Jeffreys M, et al. Survival from rectal and anal cancers in England and Wales, 19862001. Eur J Cancer 2006;42:1434-40.

31. Goggin P, Louchini R, Steben, M. Trends in anal cancer incidence in Quebec. 2005. 22nd International Papillomavirus Conference and Clinical Worshop 2005. Abstract no. P-037. 